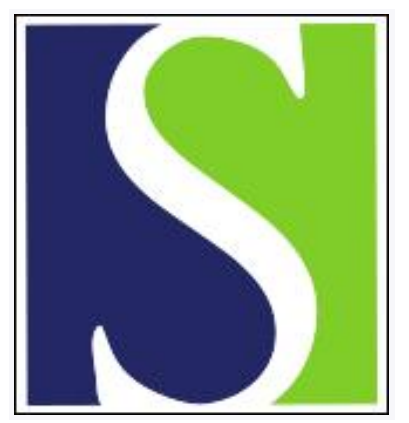

Scand J Work Environ Health 2003;29(6):478-479

https://doi.org/10.5271/sjweh.756

Issue date: Dec 2003

Dutch practice guidelines for managing adjustment disorders in occupational and primary health care

by van der Klink JJL, van Dijk FJH

Affiliation: Academic Medical Center, University of Amsterdam, Coronel Insitute, PO Box 22660, 1100 DD Amsterdam, The Netherlands. j.j.vanderklink@amc.uva.nl

The following articles refer to this text: 2008;34(1):23-32;

2010;36(6):488-498; 2013;39(2):144-154; 0;0 Special issue:0

Key terms: adjustment disorder; burnout; cognitive behavioral therapy; Denmark; discussion paper; distress; graded activity; nervous breakdown; occupational health care; practice guideline; primary health care; problem solving; stress inoculation training

This article in PubMed: www.ncbi.nlm.nih.gov/pubmed/14712856 


\title{
Dutch practice guidelines for managing adjustment disorders in occupational and primary health care
}

\author{
by Jac JL van der Klink, PhD, ${ }^{1,2}$ Frank JH van Dijk, PhD ${ }^{1}$
}

\begin{abstract}
van der Klink JJL, van Dijk FJH. Dutch practice guidelines for managing adjustment disorders in occupational and primary health care. Scand J Work Environ Health 2003;29(6):478-487.

Stress-related disorders, especially adjustment disorders, are widespread among working populations and are responsible for high costs in terms of suffering, sick leave, disability, and economic losses. Despite their high prevalence, there has been relatively little research on the effectiveness of treatments in an occupational health care setting. Guidelines for occupational physicians and general practitioners in relation to mental health problems were recently developed in The Netherlands and are discussed in this article. The guidelines provide a classification based on terms that are already used in the profession and are consistent with the diagnosis "adjustment disorder". They give recommendations for guidance and treatment on the basis of existing evidence, experience in adjacent fields, and consensus procedures. They are based on cognitive behavioral principles, mainly stress inoculation training and graded activity, and aim to enhance the problem-solving capacity of patients in relation to the work environment. The aim of this paper is to contribute to the exchange of and a discussion on methods and good practices in primary and occupational health care.
\end{abstract}

Key terms burnout, cognitive behavioral therapy, distress, graded activity, nervous breakdown, occupational health, problem solving, stress inoculation training.

In the last 20 to 30 years, occupational health professionals have been confronted with a growing number of employees suffering from various stress-related disorders (1). Many terms are used to describe these disorders, but we use "adjustment disorder" as a general concept. Adjustment disorder is an accepted medical diagnosis and is consistent with occupational health practice in that its relation to a stressor is part of the definition and marked distress or social impairment is a consequence of the disorder. Although it is hard to establish the costs in terms of absenteeism, loss of productivity, and health care consumption specific for adjustment disorders, these costs must be considerable. The International Labour Organization estimated the cost of work absence for all mental health reasons and loss of productivity at USD 200 billion a year in the United States (2). In Great Britain, about 40 million workdays are lost annually due to mental and emotional disturbances (3). In The Netherlands about half of the work disability caused by mental disorders is due to adjustment disorders.
Costs are also high for the affected person in terms of high rates of tension, anger, anxiety, depressed mood, mental fatigue, and sleep disturbances. In addition, there is a risk of being made redundant. In The Netherlands, the risk of chronic disability (after 1 year of sickness absence a person is eligible for disability benefits) was found to be higher than $20 \%$ for employees who had been on sick leave due to an adjustment disorder for 4 weeks $(4,5)$. Most of these employees did not receive any psychological or psychotherapeutic help. Apparently, it was not appreciated that these people were at risk for chronic disability.

Clearly, there is need for practical guidelines on the management of adjustment disorders for professionals who see these patients in an early phase, namely, occupational health physicians and general practitioners. However, such guidelines are difficult to develop because of confusion about diagnostics and the concepts used for stress-related disorders and because of discussion about the effectiveness of different therapeutic methods. In The Netherlands, two closely

1 Academic Medical Center, University of Amsterdam, Coronel Institute, Amsterdam, The Netherlands.

2 The Netherlands School of Public and Occupational Health (NSPOH), Amsterdam, The Netherlands.

Reprint requests to: JJL van der Klink, Academic Medical Center, University of Amsterdam, Coronel Institute, Pb 22660 , 1100 DD Amsterdam, The Netherlands. [E-mail: j.j.vanderklink@amc.uva.nl] 
related guidelines were completed recently, one for occupational health practice (6) and one combined for occupational and general practice (completed as a test version) (7). The guidelines concentrate on the diagnosis of adjustment disorders and effective strategies for client guidance or therapy. They were developed as a result of a systematic consensus process and, where possible, were evidence-based. [See the Discussion section.] Both guidelines cover diagnostic steps and steps for intervention, process evaluation, and relapse prevention. The combined guideline also covers aspects of cooperation, mutual consultation, and conduct if a difference of opinion exists between the occupational health physician and general practitioner. In this paper, we describe these Dutch guidelines on the diagnosis and therapy of adjustment disorders. Aspects such as interdisciplinary cooperation and work environment intervention are included in one or both guidelines. In this article, we concentrate on the parts of the two guidelines that are similar (ie, on the care for the individual employee). In this way, we hope to foster discussion of "best practices" and to provide support for occupational health physicians, general practitioners, occupational health psychologists, nurses, and social workers.

In the following sections, we follow the structure of the guidelines (diagnosis, intervention, monitoring, and relapse prevention) and discuss, per section, available evidence, approaches based on practice and consensus, and the way these points have been elaborated in the guidelines

\section{Diagnosis}

Search for concepts and criteria. One of the problems encountered when guidelines are developed for employees suffering from stress-related complaints is the confusing variety of terms, concepts, and diagnostic criteria used. Stress-related disorders are characterized by an identifiable stressor or stressful situation and a reaction to this situation in terms of distress symptoms, possibly combined with dysfunctioning in one or more social roles. Most occupational health physicians and general practitioners classify these disorders as stress, neurasthenia, adjustment disorders, nervous breakdown, or burnout.

Stress is a widely used term, but can be interpreted differently - as a cause, as a consequence of a process, or as the process itself. When used to indicate a disorder or a syndrome, it is usually in reference to employees who report distress symptoms, such as fatigue, irritation, and problems of concentration, but who still fulfill their occupational, social, and private roles. These people maintain control in these roles in the sense that they can keep fulfilling them, but at the cost of investing considerable energy and experiencing several complaints or symptoms.

Neurasthenia is a diagnosis in the tenth revision of the International Classification of Diseases (ICD-10) (8), but it is not present in the Diagnostic and Statistical Manual of Mental Disorders (DSM-IV) (9). It is a mental disorder characterized by chronic fatigue, weakness, and concomitant physiological symptoms.

Adjustment disorder is a diagnosis or classification in DSM IV and ICD-10 and refers to a maladaptive reaction to an identifiable stressor occurring within a short time after the onset of the stressor. It is characterized either by significant impairment in social or occupational functioning or by marked subjective distress in excess of a normal and expected reaction to the stressor (9). The symptoms often resolve within 6 months after the termination of the stressor but may persist in response to a chronic stressor.

Nervous breakdown, also denoted by the French term surmenage, is a diagnosis commonly used by general practitioners and occupational health physicians in various countries to indicate an exaggerated response to psychological stress in everyday life, especially distress symptoms, in combination with dysfunctioning (10). It is a diagnostic concept almost similar to the DSM diagnosis "adjustment disorder".

Burnout is a diagnosis that is not covered by DSMIV or ICD-10. However, since the introduction of the term in 1974 by Freudenberger (11), the term has become popular among both patients and health professionals. Maslach et al (12) characterized three dimensions of burnout. Symptoms of serious emotional exhaustion are central, and they are accompanied by either depersonalization-with a connotation that differs from that in psychiatric psychopathology (ie, related to distant feelings or cynicism in relationships with others) or reduced feelings of personal accomplishment (12).

Unfortunately, there is no consensus based on these concepts, terms, and diagnoses (originating from different periods, cultures, and conceptualizations) or on criteria that can be used in practice for employees with serious work-related problems or disorders. The term stress has so many meanings that a specific preventive or therapeutic approach cannot be based on this concept. For work-related problems, the concept of neurasthenia (ICD-10) has the major disadvantage that it is a description at a symptom level and is not associated with stressful situations or with social dysfunctioning (work or private life). The diagnosis "adjustment disorder" is an accepted medical diagnosis and is consistent with experience in occupational health practice. The relation to a stressor is part of the definition, and marked distress and social impairment are consequences of the disorder. However, occupational physicians prefer diagnostic 
terms that enable them to differentiate between employees who are on sick leave and those who are not, especially because the main risk of these disorders lies in the development of prolonged disability. The term "nervous breakdown" (or its synonym surmenage) focuses on employees who have suffered such a loss of control that they are on sick leave, and therefore it is commonly used by occupational health physicians. In contrast, the term "burnout" covers symptoms that are focused on specific problems with performance at work, such as cynicism and experience of professional ineffectiveness. The term "burnout" is used both for the final stage of a process and for the process itself. Employees in a burnout process are often not yet severely impaired in their social roles, and many are still at work, although not without minor or major problems.

The diagnosis "adjustment disorder", which includes impaired functioning and marked distress, seems most appropriate because it covers the work-related problems seen in daily practice. However, in occupational health practice a differentiation between employees still at work and those on sick leave is considered important, as has already been mentioned. Moreover, occupational health physicians differentiate in practice between a category of patients with a short case history (less than 3 months) of exposure to stressors and a favorable prognosis and patients with a history much longer than 3 months and a relatively unfavorable prognosis.

Classification in guidelines. In accordance with the arguments already discussed, the Dutch guidelines use the general concept of adjustment disorders (stress-related disorders) with subclassification into the categories of distress, nervous breakdown, and burnout.

Distress narrows the broad concept of stress to the subjective reaction pattern to a stressor. Emphasis is given to the aspect that functioning is mainly intact; people maintain control over their interaction with the situation and still go to work.

Nervous breakdown emphasizes the impairment in social or occupational functioning (ie, loss of control over the situation within a period of 3 months after the stressor). Patients have to drop several roles, particularly demanding roles. Work is one of the first roles to be sacrificed, sick leave being a probable consequence. Marked subjective distress is an accompanying problem in most cases.

Burnout is characterized by emotional exhaustion. In addition either depersonalization, as defined earlier, or the experience of ineffectiveness must be present. Emphasis is placed on the long duration that distinguishes burnout from nervous breakdown; usually, there is more than 1 year between the onset of the chronic stressor and the ultimate crisis.

Adjustment disorders should be distinguished from conditions in which people have problems or a strong emotion (eg, anger in case of a conflict) without significant distress in excess of a normal and expected reaction and also from more severe disorders. Distress symptoms in combination with an identifiable stressor can occur together with bereavement and various psychiatric disorders. Thus the diagnosis of adjustment disorder should not be used when the disturbance meets the criteria of another DSM disorder, such as a specific anxiety or mood disorder, and not when the symptoms represent bereavement. In particular, it is important that occupational health care and primary health care professionals identify depression and anxiety disorders (among them posttraumatic stress disorders). Both disorders are characterized by a high prevalence, overlap with nonspecific distress symptoms (table 1), and a potential need for specific care (specific therapeutic protocols) in addition to guidance focused on the stress-related part of the disorders. A few specific criteria can

Table 1. Symptoms related to depression and anxiety. ${ }^{\mathrm{a}}$

\begin{tabular}{lll}
\hline Disorder & Specific symptoms $^{b}$ & Nonspecific symptoms ${ }^{c}$ \\
\hline Depression & Loss of pleasure in almost all activities & Dysphoric mood \\
& Marked psychomotor agitation & Loss of interest \\
& Feelings of worthlessness, self-reproach or excessive or & Sleeping problems (insomnia or hypersomnia) \\
& inappropriate guilt & Loss of energy, fatigue \\
& Suicidal ideation & Diminished ability to concentrate, slowed thinking, indecisiveness \\
Marked weight loss or gain & Marked changes in appetite \\
& Continuous anxious mood or excessive worrying for & Anxious mood \\
& at least 1 month & Worrying \\
& Persistent and irrational fear & Mild avoidance \\
& Recurrent panic attacks & Palpitations, trembling, shaking, sweating, dizziness, \\
Compelling desire to avoid & other physical symptoms \\
& Obsessions or compulsions & \\
\hline
\end{tabular}

\footnotetext{
a For the diagnosis one should apply the criteria of the American Psychiatric Association (9).

${ }^{b}$ Specific symptoms are more exclusive to the disorder in question.

${ }^{c}$ Nonspecific symptoms common with those often experienced by patients with a stress-related disorder.
} 
be indicated for both disorders (table 1). Depression is characterized by depressed cognitions and vital symptoms and by severe anhedonia, whereas anxiety disorder is characterized by excessive anxiety or concern.

The Dutch guidelines use the following classification for occupational and primary health care populations:

\author{
- Adjustment disorders \\ - Distress \\ - Nervous breakdown \\ - Burnout \\ - Depression \\ - Anxiety \\ - Remaining psychiatric disorders.
}

\section{Intervention}

Search for evidence. As the first step in providing a basis for the development of an intervention, searches were made for intervention and prognostic studies that might reveal populations at risk. A Medline search on workrelated adjustment disorders ${ }^{3}$ failed to identify any controlled intervention studies. This failure indicated that, despite the high prevalence of adjustment disorders, little is known about the effectiveness of treatments (13).

In the adjacent domain of preventive programs about 50 controlled studies of preventive, or stress management, programs were identified, mostly aimed at preventing problems with performance, sickness absence, or permanent work disability. ${ }^{4}$ The conclusion of several qualitative and quantitative reviews $(1,14-16)$ was that stress management interventions are effective in reducing the negative aspects of stress. One of these, a recent meta-analysis (14), concluded that cognitive behavioral and multimodal (cognitive behavioral interventions combined with relaxation techniques) interventions appear to be the most effective. Relaxation techniques also proved to be effective, although less than the programs with a cognitive approach. In terms of outcome variables, the meta-analysis showed that cognitive behavioral intervention can improve perceived quality of worklife, enhance psychological resources and responses, and reduce symptoms. In terms of psychophysiological outcomes, relaxation techniques, whether pure or em- bedded in a multimodal program, appear to be effective.

No randomized controlled studies were found that evaluated the therapy or guidance of employees with burnout, but several controlled studies evaluated intervention among a working population with a burnout measure as the dependent variable (17-25). However, none of the studies concerned patients diagnosed with burnout. Cognitive programs appeared to be the most effective.

A search of Medline and Psycinfo ${ }^{5}$ for prognostic studies of adjustment disorder did not detect any studies published in peer-reviewed journals.

\section{Search for approaches}

A search of Medline, Psycinfo, and Clearinghouse National Guidelines (in the United States) for guidelines on the diagnosis and therapy of adjustment disorders identified a few publications with an unclear status. They were either written by a single author, or the development procedure was not clarified. This lack of publications contrasts with the existence of clinical guidelines, for example, for major depressive disorder (26, 27).

Some of the publications retrieved concentrated on more general topics, for instance, prevention of disability (28), or on related areas, for example, guidelines for brief psychodynamic psychotherapy (29), a guideline for family physicians for investigating fatigue of less than 6 months' duration (30), and a document on the recognition, prevention, and remediation of burnout among health care professionals participating in the care of children with cancer (31). In general, these articles drew the same conclusions as the reviews and meta-analyses of stress management, namely, that cognitive therapy, stepwise reintegration planning, and relaxation training are effective forms of psychotherapeutic intervention.

The lack of well-designed studies means that there is no high-level evidence for any type of intervention for work-related adjustment disorders. Practice-based or consensus-based guidelines have not been published. Nevertheless, there are sound arguments that therapy needs to be supportive, active, flexible, and goal-directed and it should work within a time-limited context (32-34). Therapy uses encouragement, supports the

All searches conducted in the process of guideline development were repeated for this article to obtain a timely overview. The outcomes of the two searches were consistent. We searched for articles published from 1980 on and concerning "adjustment disorders" combined with two groups of terms, one related to the population (employee, occupational, vocational, rehabilitation, work, job, absenteeism, and sick leave) and the other related to intervention (therapy, treatment, protocol, program, intervention, primary care, prevention, and employee assistance program). For the search terms see van der Klink et al (14).

5 From 1980; terms were adjustment disorder and prognosis/prognos*. 
patients' strengths, and minimizes or plays down past problems. Education and information play an important role. Therapy, according to these principles, enables patients to develop a realistic attitude towards their situation, to realize that they are not alone with their problems, and to become aware of useful and helpful resources (32). Because the impairment of social or occupational functioning is a central feature of work-related adjustment disorders, caused by a temporary lack of control over interaction with the situation, therapy and guidance concentrate on helping patients to regain control and to rebuild their social and occupational contacts and activities. This activity is especially important when the main risk is that the impairment becomes chronic because the patient does not regain his tasks and social and occupational roles. There are several methods of proved effectiveness that focus on the patient's regaining control and activities, for instance, stress inoculation training, cognitive restructuring, graded activity, and time contingency (table 2).

Cognitive-behavioral intervention, based on cognitive restructuring, has proved effective in both preventive programs $(14,16)$ and curative programs (37). The same is true for stress inoculation training (38). A graded activity approach based on time contingency has proved effective with patients with lowback pain $(39,40)$. Early efforts to help the patient regain function appear to be effective (28). These principles have been applied in the Dutch guidelines for the diagnosis and treatment of adjustment disorders $(6,7)$.

Three-phase model, a basic scheme for the guidelines. In the guidelines, regaining control and resuming earlier activities are regarded as active processes in which three phases can be distinguished. The first phase is dominated by the crisis. Patients are often "out of joint" or disorganized. They are tired, and sometimes they have no clear insight into what has happened. Regaining control in this phase means understanding what has happened and coping emotionally with it, therefore obtaining understanding and acceptance. In the second phase, patients have to gain insight into the stressors that contributed to their distress and into possible solutions. The acquisition of skills can be part of this phase. In the third phase, the solutions and skills that have been acquired have to be applied and put into practice in the social and occupational roles that patients have dropped.

This three-phase model is based on experience gained with patients with successful recovery. The three phases together resemble the phases in stress-inoculation training. As in grief therapy, the therapeutic approach aims at guiding patients for whom recovery has stagnated. To prevent a mere passive perception of the recovery process, the phases are described to patients as tasks (41). These tasks are to acquire insight and acceptance and to draw up an inventory of stressors and solutions. Subsequent tasks are to apply the solutions and learned skills and to resume social roles and return to work.

Occupational health physicians and general practitioners have two roles to fulfill. They must monitor the recovery process, by ascertaining that patients fulfill the recovery tasks, and they must intervene when necessary. Who performs which role depends, among other things, on whether a patient's problem is mainly work-related or not.

Interventions elaborated in the guidelines. Intervention is needed when the aforementioned tasks are not fulfilled spontaneously. The aim of all intervention is to enable patients to complete the recovery tasks and to increase their problem-solving capacity. Although assignments are given, it is important that patients do not receive a prescription defining how they should solve their problems, because such a prescription would

Table 2. Central features of stress inoculation training, cognitive restructuring, graded activity, and time contingency.

\begin{tabular}{ll}
\hline Method & Characteristic \\
\hline Stress inoculation training & $\begin{array}{l}\text { Designed to train effective coping skills to enhance resistance to stress }(35,36) \\
\text { Encompasses three-stage intervention of (i) education with a goal of helping the patient better understand the nature of } \\
\text { stress and stress effects, (ii) skill acquisition, encouraging the patient to develop and practice a repertoire of coping } \\
\text { skills, and (iii) application of coping skills in increasingly challenging situations }\end{array}$ \\
& $\begin{array}{l}\text { Aimed at the patient's changing thoughts (cognitions) to help him or her develop a different, more productive point of } \\
\text { view of his or her problems } \\
\text { Cognitive restructuring } \\
\text { Creates a new starting point from which solutions become apparent } \\
\text { Helps identify irrational thoughts or beliefs that block a more distant, productive view and helps replace irrational beliefs } \\
\text { with more rational cognitions } \\
\text { Uses a time contingent scheme to resume activities gradually } \\
\text { Implies that activities are built up according to a prestructured time schedule and is thus not dependent on the course } \\
\text { of symptoms } \\
\text { Avoids the pitfall of the sequence "I can only start doing this or that when my fatigue (or any other symptom) is less." }\end{array}$ \\
\hline
\end{tabular}


encourage dependence rather than independence and discourage an active approach by the patient. Thus intervention aims to keep the patient moving on the road to recovery (ie, to regaining control) not to take him by the hand (and take over control).

Intervention in the crisis and understanding phase aims at supporting the acquisition of insight and the acceptance of what has happened. The elements are education about stress, stress responses and adjustment disorders, provision of a rationale, a realistic prospect, positive re-labeling, and a schedule for various nondemanding activities. These interventions can help patients regain control at a cognitive and emotional level. Providing patients with adjustment disorders with a rationale for the problems they experience is a powerful form of intervention, especially when given as a metaphor. A rationale should offer a plausible explanation of the situation a patient has encountered and should provide a favorable perspective of the problem. In this manner, the rationale that all psychopathology has its roots in the past (ie, in one's youth) offers an explanation but also implies an unfavorable perspective because one cannot change one's youth. The rationale that most psychopathology is a result of a combination of a person's situation, perception, thoughts, and behavior is favorable because people can choose to influence the stressful situation and their perceptions, thoughts, or behavior, depending on the perceived controllability. A broadly applicable rationale for adjustment disorders is the balance metaphor (figure 1).

The balance metaphor illustrates that there should be an equilibrium between the load to bear and a person's ability to bear the load. When this equilibrium is disturbed, either the load should be-temporarily-reduced (fewer workhours, fewer other obligations, some problems solved, effective time management) or the ability to bear should be increased (a rationale, problemsolving attitude, effective relaxation, physical training,
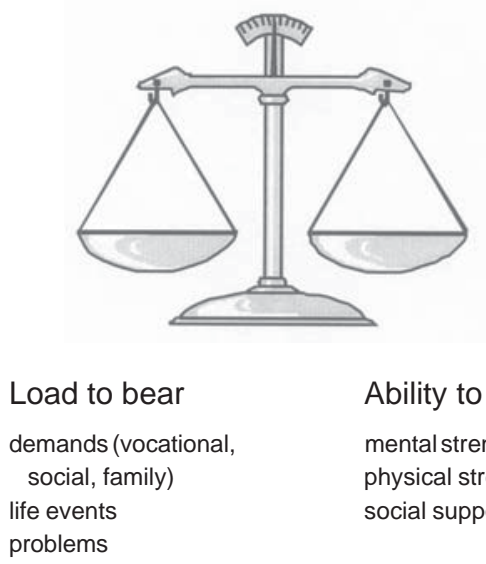

Ability to bear

mental strength physical strength social support

Figure 1. The balance metaphor.

mobilization of social support, enlargement of decision latitude). The phase model, explained in terms of tasks, can serve as a rationale for the recovery process.

Intervention in the insight phase aims to support the making of an inventory of problems or stressors and consequently of possible solutions. It consists of such undertakings as a worrying assignment, a stressor and solution inventory, a writing assignment, and decision protocol.

The stressor and solution inventory are at the core of this phase. The accumulation of demands and problems often leads to a diffuse feeling of distress and threat and to avoidance of an active approach to problems. Drawing up an inventory of stressors can empower patients and make them feel more in command of the situation (42). For this reason, patients are asked to draw up a list of seven to ten stressors in any aspect of their lives (work, social, etc). These stressors must be concrete, not vague (such as, "my work", or "atmosphere at work"). Patients are then asked to indicate to what extent each stressor can be influenced ("Can it be influenced immediately?", "Is a strategy or other people needed?" or "Can it not be influenced?"). Then, patients design strategies to manipulate the stressors, starting with those that lend themselves to an immediate approach and then proceeding to those that may need a more complex strategy or the help of others. Finally, patients learn to accept stressors that cannot be influenced.

Intervention in the rehabilitation phase aims at helping patients apply the strategies and solutions they have worked out and at building up activities and roles that they have dropped. The topics are "rehabilitation schemes" and "anticipation of difficulties and drawbacks".

The essence of this phase is that patients work on a time-contingent basis, after setting realistic goals for their rehabilitation. Therefore, resolving problems and resuming earlier activities do not depend on the course of symptoms. The main danger, that patients develop a chronic disorder, lies in the fact that symptoms such as fatigue deter patients from taking up social and occupational roles. By asking patients to make two schemes, one that is maximally demanding or challenging and one that is maximally safe, a range is created in which both the patient and professional can determine a recovery trajectory, mostly positioned somewhere in between. This flexible approach allows patients to speed up or slow down within the defined range in response to problems encountered during rehabilitation.

\section{Monitoring the process and relapse prevention}

The analysis of cases in which stress responses have become chronic shows that often little is done to correct or adjust a patient's progress. In many instances patients are encouraged to rest and are given comforting counseling as guidance. The Dutch guidelines 
provide an active monitoring strategy for the regular evaluation of the recovery process in order to prevent cases from becoming chronic.

The evaluation criteria are based on the three phases described in the preceding text. There are four evaluation moments (table 3 ): the first at the end of the first phase, the second halfway through the second phase, the third at the end of the second phase, and the fourth at the end of the third phase. For persons' diagnosed with a nervous breakdown, these evaluation moments fall at 3 -week intervals. Thus after 3 weeks the tasks of the first phase should have been completed, and the criteria described for the first evaluation moment should have been met. The timing of the evaluation moments is not absolute but, instead, serves to warn professionals that recovery may be slow and that a patient may be at risk of developing a chronic problem. Minimal intervention can be used to support patients in fulfilling the tasks of a given phase. If there is a persistent stagnation of the patient's progress, a thorough evaluation should take place to identify the underlying cause, and the patient should be referred to more specialized care, if warranted. Although the periods are predominantly practice based, there is evidence that the recovery process can be maximized at about 12 weeks. In a recent intervention study, $96 \%$ of the patients who had received activating intervention had resumed their work totally or partly within 12 weeks (13). In many cases, it will take a considerably shorter period.

The same scheme can be applied to the work situation, and, in such a situation, management and supervisors may play a decisive role. Tasks are set that correspond with those of patients, namely, identifying the problem, developing a solution to the problem, and finally applying the solution. Occupational health physicians, occupational health nurses, psychologists, or social workers can coach management and colleagues in this process.

Because the recovery process is also a learning process in which problem-solving skills are acquired, relapse prevention is an important aspect of therapy. The aim of relapse prevention is to safeguard the results of the recovery and learning processes and to prevent a downward spiral if patients relapse. It increases the likelihood that patients will react adequately to new or recurrent problems. Thus the occupational health physician or general practitioner involved should check whether patients recognize early signals (feelings, mood, cognition, behavior) and whether the (work) environment also recognizes these signals. They should also ensure that patients are sufficiently aware of which situations, attitudes, and cognitions play a role in the development of adjustment disorders. Finally, the occupational health physician or general practitioner should attempt to determine how patients and the (work) environment will react if signals or situations return.

\section{Discussion}

In this article, we have described Dutch guidelines on adjustment disorders for occupational health physicians and general practitioners. Adjustment disorders were subdivided into distress, nervous breakdown, and burnout. The guidelines are based on an activating approach and incorporate process evaluation.

In this discussion section, we focus first on the validity of the method described, and then on the feasibility and relevance of these guidelines to occupational health care and general practice. Finally, we comment on cultural aspects.

There is little published research that evaluates the effectiveness of intervention for work-related adjustment disorders with methodological rigor. A protocol similar to the guidelines described here proved to be highly effective in the prevention of long-term sickness absence and in relapse prevention (13). Further evaluation is necessary to provide additional scientific evidence and to test new approaches. Randomized clinical trials and other well-designed studies are needed as a foundation for evidence-based practice. However, because of the meticulous consensus and review procedures used in the development of these guidelines, they are firmly practice- and consensus-based. To be eligible for

Table 3. Tasks to be accomplished in the three phases of recovery and the schedule for the evaluation and its criteria.

\begin{tabular}{|c|c|c|c|}
\hline Recovery phase & Tasks to accomplish & $\begin{array}{l}\text { Evaluation } \\
\text { time }\end{array}$ & Criteria for advancement or change-over \\
\hline $\begin{array}{l}\text { 1. Crisis and under- } \\
\text { standing }\end{array}$ & $\begin{array}{l}\text { Rest, insight and } \\
\text { acceptance }\end{array}$ & I. End of phase 1 & $\begin{array}{l}\text { I. The patient has a rationale for the situation or accepts one and has a problem- } \\
\text { oriented attitude; preoccupation in thinking shifts from consequences such as } \\
\text { symptoms to causes such as problems and stressors }\end{array}$ \\
\hline 2. Insight & $\begin{array}{l}\text { Defining the problem } \\
\text { and making an inven- } \\
\text { tory of stressors }\end{array}$ & $\begin{array}{l}\text { II. Halfway through } \\
\text { phase } 2 \\
\text { III. End of phase } 2\end{array}$ & $\begin{array}{l}\text { II. The patient has a problem-solving attitude and is open to the suggestion to } \\
\text { make an inventory of possible solutions } \\
\text { III. The patient has an application-oriented attitude and is open to the } \\
\text { suggestion to apply strategies and to build up demanding activities }\end{array}$ \\
\hline 3. Rehabilitation & $\begin{array}{l}\text { Working out strategies, } \\
\text { rehabilitation }\end{array}$ & IV. End of phase 3 & $\begin{array}{l}\text { IV. Roles that have been dropped during the crisis are (at least partially) } \\
\text { resumed }\end{array}$ \\
\hline
\end{tabular}


authorization, guidelines for occupational health physicians should be developed according to the following procedure: (i) after a review of the relevant literature and consensus meetings, a project team of occupational health physicians should prepare a first draft, (ii) this draft should be reviewed by experts from inside and outside the field of occupational medicine, (iii) a revised draft should be judged and tested by practicing occupational health physicians, and (iv) a final version should be submitted to the guideline committee for authorization.

The present guidelines were prepared by a project team of 21 occupational health physicians and 1 psychologist. The first draft was reviewed by 15 experts (among others, occupational health physicians, psychiatrists, general practitioners, and psychologists). The second draft was tested and commented on by 21 practicing occupational health physicians, selected at random from the files of the Dutch Association of Occupational Physicians.

Despite this thorough procedure, scientific evidence is the most solid and preferable base for medical practice. The evidence for these guidelines is based on only one randomized clinical trial (13). Other randomized studies are currently being conducted. It has been a conscious choice of the Dutch Association of Occupational Physicians to develop these guidelines at this stage, primarily because of the high individual and societal costs of disability due to adjustment disorders and the unsatisfactory results of practice in force.

Other good practices may exist that probably share some core elements with the Dutch guidelines, such as activation and graded activity. Where our focus was cognitive behavioral, effective methods could also be based on relaxation techniques and physical activation or on intervention focusing on work organization. This article may contribute to a discussion and exchange of good practices on this topic.

The three-phase model provides a structure to support patients in fulfilling their recovery tasks. The interventions described are primary care interventions for occupational health care professionals or general practitioners and may aid this process. If these interventions insufficiently stimulate progress, then the case should be thoroughly evaluated to detect underlying causes for the stagnation of the recovery. This evaluation may lead to work-oriented intervention or reveal patient-related aspects that go beyond the scope of the coping-enforcing interventions that generalists without a specialized therapeutic background can conduct. The patient should then be referred to a specialist. Thus process monitoring is an instrument for the professional generalist that guarantees that the intervention is as basic as possible but as specialized as required.
Patients with distress can follow the same schedule as that described in this paper for patients with "nervous breakdown", on the understanding that social or occupational roles do not have to be assumed in the third phase. For patients with burnout, who usually have a long history, the duration of the three phases may be longer. In such cases, the scheme can help occupational health physicians and general practitioners to distinguish between patients with burnout who can be helped by the method as described in this paper and those who need to be referred for more specialized help and intervention.

A study among occupational health physicians revealed that the guidelines have a high rate of acceptance among occupational health physicians and that the attitude and the intention to use them are positive. However, implementation of the guidelines lags behind their high acceptance. On a 7-item scale with a 5-point Likert scale for each item, 1 representing negative and 5 positive, the mean score for positive attitude was 4 . On a 2-item scale for application of the guidelines, with a 5-point Likert scale for each item ranging from 1 for never to 5 for always, the mean score was 2.5 (43). A study among occupational health physicians and general practitioners trained in the use of guidelines for concerted action on mental health problems showed high use of and satisfaction with the guidelines by both professional groups (44). These outcomes indicate that further implementation efforts do not need to focus on increasing the acceptance of the guidelines but, instead, on training professionals in their use.

As we have already mentioned, the schedule for recovery is not absolute and is strongly influenced by cultural factors. Occupational health physicians trained in the use of the guidelines and working in the Dutch Caribbean commented that, while the guidelines were valid for the population, the schedule for recovery was much shorter than that given in the scheme. The type of work involved may also play a role in this difference. Another potential cultural influence involves the coping strategy used. The guidelines emphasize active coping, the dominant coping strategy used in Western societies. However, it is not known whether this focus will be equally effective in societies or (sub)cultures with a greater emphasis on passive coping strategies. In the aforementioned meta-analysis (14), only 2 of 48 studies were conducted in a non-Western society $(45,46)$. One study evaluated a cognitive behavioral approach, and the other a relaxation-based intervention, which is more directed towards passive coping. Both interventions proved to be effective. In this sample of two, the cognitive behavioral intervention was more effective, but no conclusions can be drawn from this result.

Work stress has both individual and organizational aspects. Both aspects are valid and essential and should 
be taken into account in prevention, diagnostics, therapy, and rehabilitation $(1,3)$. The Dutch guidelines for occupational health physicians and general practitioners encompass organizational diagnostics and some basic interventions, especially contact with management, although the emphasis is on patient guidance. In the future this individual approach will be complemented with a worksite approach, focused on, for example, management style, community building, and conflict solving. For pragmatic reasons we have limited our focus to patient guidance in this article.

\section{Concluding remarks}

The relatively mild diagnosis "adjustment disorder" has far-reaching disabling consequences for those suffering from it in The Netherlands and other countries. Current occupational health and general practice approaches are often not successful in preventing the consequences of these disorders. Evidence-based methods are available in related fields. The guidelines discussed in this article were developed in a consensus procedure, and they are based on evidence where possible, on good practice experience, and on existing approaches with proved effectiveness in related fields. Given the paucity of high-level evidence in this area and the complexity of the issue, some choices were predominantly based on consensus; other solutions may also be possible and potentially effective. The acceptance of the guidelines is high among professionals, but their actual use is in the implementation phase. The guidelines appear to be effective when physicians are trained in their use, but further evaluation is needed. We recommend an exchange of methods and good practices to support professionals and to challenge researchers to conduct more evaluation studies.

\section{Acknowledgments}

Grants were obtained from The Netherlands School of Public and Occupational Health (NSPOH).

\section{References}

1. Murphy LR. Stress management in working settings: a critical review of the health effects. Am J Health Promot 1996;11:112-35.

2. International Labour Organization. Job stress: The 20th century disease. Geneva: United Nations Labour Office, 1993. World labour report, no 6.

3. Cox T, Griffiths D, Rial-Conzález E, editors. European Agency for Safety and Health at Work: research on work-related stress. Luxembourg: Office for Official Publications of the European Communities; 2000.

4. Schröer, CAP. Verzuim wegens overspanning: een onderzoek naar de aard van overspanning, de hulpverlening en het verzuimbeloop [Absenteeism due to 'overstrain': a study of the nature of overstrain, therapeutic assistance and absenteeism] [dissertion]. Maastricht: Universitaire Pers; 1993. English summary.

5. Schröer, CAP. De toename van arveidsongeschiktheid wegens psychische aandoeningen [The increase of work disability due to mental disorders]. Tijdschr Bedr Verzekeringsgeneesk 1997;5:16-24.

6. van der Klink JJL, editor. Handelen van de bedrijifsarts bij werknemers met psychische klachten [Guideline for occupational physicians for mental disorders]. Eindhoven: NVAB (Dutch Association of Occupational Physicians); 2000.

7. van der Klink JJL, editor. Eerstelijns handelen bij psychische klachten en arbeid: richtlijn voor huisartsen en bedrijfsartsen: testversie [Mental disorders and work: guideline for general practitioners and occupational physicians: test version]. Utrecht/Amsterdam: KNMG/SKB; 2000.

8. World Health Organization (WHO). The ICD-10 classification of mental and behavioural disorders: clinical descriptions and diagnostic guidelines. Geneva: WHO; 1992.

9. American Psychiatric Association. Diagnostic and statistical manual of mental disorders (DSM-IV. 4th ed. Washington (DC): American Psychiatric Press; 1994/2000.

10. Terluin B. Overspanning onderbouwd: een onderzoek naar de diagnose surmenage in de huisartspraktijk [Nervous breakdown substantiated: a study of the general practitioner's diagnosis of surmenage][dissertation]. Utrecht: Detam; 1994. English summary.

11. Freudenberger HJ. Staff burnout. J Soc Issues 1974;30:169-5

12. Maslach C, Schaufeli WB, Leiter MP. Job burnout. Annu Rev Psychol 2001;52:397-422.

13. van der Klink JJL, Blonk RWB, Schene AH, van Dijk FJH. Reducing long-term sickness absence by an activating intervention in adjustment disorders: a cluster randomised controlled design. Occup Environ Med 2003;60:429-37

14. van der Klink JJL, Blonk RWB, Schene AH, van Dijk FJH. The benefits of interventions for work related stress. Am J Public Health 2001;91:270-6.

15. Murphy LR. Occupational stress management: a review and appraisal. J Occup Psychol 1984;57:1-15.

16. Bamberg E, Busch C. Betriebliche Gesundheitsförderung durch Stressmanagementtraining: eine Meaanalyse (quasi-)experimenteller Studien [Employee health improvement by stress management training: a meta-analysis of (quasi-) experimental studies]. Z Arb Organisationspsychol 1996;40: 127-37.

17. Brown L, Mutual help staff groups to manage work stress. Soc Work Groups 1984;7:55-66.

18. Corcoran KJ, Brice AK. Interventions in the experience of burnout: effects of skill development. J Soc Serv Res 1983;7: 71-9.

19. van Dierendonck D, Schaufeli WB, Buunk BP. The evaluation of an Individual burnout intervention program: the role of inequity and social support. J Appl Psychol 1998;83:392-407.

20. Freedy JR, Hobfoll SE. Stress inoculation for reduction of burnout: a conservation of resources approach. Anxiety Stress Coping 1994;6:311-25.

21. Higgins NC. Occupational stress and working women: the effectiveness of two stress reduction programs. J Vocat Behav 1986;29:66-78.

22. Larson DG. Developing effective hospice staff support groups: pilot test of an innovative training program. Hospice J 1986;2:41-5.

23. Pines A, Aronson, E. Career burnout: causes and cures. New York (NY): Free Press; 1988. 
24. Schaufeli WB. The evaluation of a burnout workshop for community nurses. J Health Hum Resour Adm 1995;18:1140 .

25. West DJ, Horan JJ, Games PA. Component analysis of occupational stress inoculation applied to registered nurses in an acute care hospital setting. J Couns Psychol 1984;31:209-18.

26. American Psychiatric Association. Practice guideline for the treatment of patients with major depressive disorder (1993, revised 2000). Am J Psychiatry 2000;157 4 Suppl:1-45.

27. Marwijk HWJ, Grundmeijer HGLM, Brueren MM, Sigling HO, Stolk J, van Gelderen MG, et al. NHG-standaard depressie [Dutch association of family physicians guideline for depression]. Huisarts Wet 1994;37;482-90.

28. McGrail MP, Lohman W, Gorman R. Disability prevention principles in the primary care office. Am Fam Physician 2001; 63:679-84.

29. Horowitz MJ. Stress-response syndromes: a review of posttraumatic and adjustment disorders. Hosp Community Psychiatry 1986;37:241-9.

30. Godwin M, Delva D, Miller K, Molson J, Hobbs N, MacDonald S, MacLeod C. Investigating fatigue of less than 6 months' duration. Can Fam Physician 1999;45:373-9.

31. Spinetta JJ, Jankovic MJ, Ben Arush MW, Eden T, Epelman $\mathrm{C}$, et al. Guidelines for the recognition, prevention, and remediation of burnout in health care professionals participating in the care of children with cancer: report of the SIOP Working Committee on Psychosocial Issues in Pediatric Oncology. Med Pediatr Oncol 2000;35:122-5.

32. Carlson J. Adjustment disorders and V codes. In: Sperry L, Carlson J. Psychopathology and psychotherapy: from DSMIV diagnosis to treatment. Washington: Taylor \& Francis; 1996.

33. Maxmen JS. Essential psychopathology. New York(NY): WW Norton; 1986.

34. Sperry L, editor. Varieties of brief therapy. Individ Psychol 1989;45(1/2 special issue).

35. Meichenbaum DH, Cameron R. Stress inoculation training. In: Meichenbaum DH, Jarenko ME, editors. Stress reduction and prevention. New York (NY): Plenum Press;1983.

36. Meichenbaum DH. Stress inoculation training: a twenty-year update. In Woolfolk RL, Lehrer PM, editiors. Principles and practice of stress management. 2nd ed. New York (NY):
Guilford Press; 1993.

37. Firth J, Shapiro DA. An evaluation of psychotherapy for jobrelated distress. J Occup Psychol 1986;59:111-9.

38. Saunders T, Driskell JE, Hall Johnston J, Salas E. The effect of stress inoculation training on anxiety and performance. $J$ Occup Psychol 1997;70:170-86.

39. Lindström I, Ohlund C, Eek C, Wallin L, Peterson LE, Fordyce WE, et al. The effect of graded activity on patients with subacute low back pain: a randomized prospective clinical study with an operant conditioning behavioral therapy approach. Phys Ther 1992;72:279-90; discussion 291-3.

40. Lindström I, Ohlund C, Eek C, Wallin L, Peterson LE, Nachemson AL. Mobility, strength, and fitness after a graded activity for patients with subacute low back pain: a randomized prospective clinical study with a behavioral therapy approach. Spine 1992;17: 641-52.

41. Worden JW. Grief counselling and grief therapy. London: Tavistock; 1983.

42. Fontana D. Managing stress, problems in practice. London: British Psychological Society; 1989.

43. Heinemans AMEC, Rebergen DS. Burnout in de praktijk: een onderzoek naar de invloed van de werkveleving van bedrijfsartsen op hun begeleiding van werknemers met psychische klachten [Burnout in practice: a study on the influence of the perception of quality of work by occupational physicians on their guidance of employees with mental problems]. Utrecht: University of Utrecht; 2002.

44. Hulshof CTJ, Broersen JPJ, de Haan S. Samenwerkingsrichtlijn eerstelijns handelen bij psychische klachten en arbeid evalatie van fase 2: praktijktest [Evaluation report on the practice of the guideline for concerted action on mental health problems by GP's and OP's]. Amsterdam: SKB; 2002.

45. Lee S, Swanson Crockett M. Effect of assertiveness training on levels of stress and assertiveness experienced by nurses in Taiwan, Republic of China. Issues Ment Health Nurs 1994;15:419-32.

46. Tsai S, Swanson Crockett M. Effects of relaxation training, combining imagery, and meditation on the stress level of Chinese nurses working in modern hospitals in Taiwan. Issues Ment Health Nurs 1993;14: 51-66.

Received for publication: 23 July 2002 